\title{
Role of Anopheles baimaii: potential vector of epidemic outbreak in Tripura, North-east India
}

\author{
Nilanju Pran Sarmah¹, Ipshita Pal Bhowmik¹, Devojit Kumar Sarma ', Chandra Kanta Sharma1, \\ Gajendra Kumar Medhi ${ }^{1}$, Pradyumno Kishore Mohapatra ${ }^{1}$, Jagadish Mahanta ${ }^{1}$, Dibya Ranjan Bhattacharyya ${ }^{1}$ \\ ${ }^{1}$ ICMR-Regional Medical Research Centre, NE (Indian Council of Medical Research), Dibrugarh, Assam, India \\ Keywords: global health \\ https://doi.org/10.29392/joghr.3.e2019036
}

\section{Journal of Global Health Reports}

Vol. 3, 2019

\begin{abstract}
Background
Malaria is one of the foremost public health concerns in north-east India. The state of Tripura, belonging to north-east India recently reported an outbreak of malaria during 2014 with thousands of positive cases and several deaths due to malaria. The epidemic has occurred mainly in the high transmission areas affecting the young population. This study was undertaken to find out the vector species responsible for the outbreak.
\end{abstract}

\begin{abstract}
Methodology
House frequenting female anophelines were collected by using CDC (Centers for Disease Control and Prevention) miniature light traps. Species confirmation of Anopheles (An.) baimaii and An. minimus and presence of malaria parasite in mosquitoes was done by rDNA based molecular identification method.
\end{abstract}

\section{Results}

A rapid entomological survey revealed the involvement of An. baimaii on the basis of evidences like high salivary gland infectivity to P. falciparum (11\%), dominant presence over other anophelines and exclusive anthropophily, detected during the epidemic.

\section{Conclusion}

This is the first report of incrimination of An. baimaii as vector of malaria in Tripura. Encroachment of dense forested areas for shifting cultivation (Jhum) and other activities, suitable environmental condition for vector propagation, and other factors like inadequate vector control and surveillance in remote areas seems to be the probable causes responsible for this epidemic.

With only $3.7 \%$ of the Indian population, North-East (NE) India has been contributing 8-12\% malaria, 10-20\% Plasmodium falciparum (P. falciparum) cases and about $14.78 \%$ of deaths due to malaria in the country's average. ${ }^{1}$ North-East region of India comprises of eight states of which Tripura is considered to be the smallest with a population of 3.679 million (Census 2011). Tripura is known to be endemic for malaria with preponderance of $P$. falciparum. ${ }^{1}$ The distribution of the disease is not uniform in Tripura and shows wide variation both spatially as well as temporarily. However, frequent outbreaks of malaria in different states of NE region prior to 2008 used to be very common. ${ }^{2-5}$ In recent times, the outbreak of malaria has been decling due to large scale introduction of artemisinin based combination therapy as well as wide spread use of insecticide treated bed nets (ITBN) and LLIN (Long lasting insecticide treated bed nets) distribution along with indoor residual spray (IRS). However, an exception occurred in 2014 in the form of a severe malaria outbreak in Tripura leading to high morbidity and mortality. Historically, the outbreak of malaria in NE India is attributed by Anopheles minimus (An. minimus) and usually limited to or start from foot hill area. Till date, An. baimaii is found to maintain high endemicity of malaria in stable malaria condition. In tribal areas of NE states malaria epidemics are rare. As the information on role of the malaria vectors in Tripura are limited and earlier studies reported the presence of An. minimus confirming its role in malaria transmission in Tripura. We carried out an entomological investigation to find out the role of different vectors of malaria in the epidemic of Tripura and found An. baimaii to be the vector responsible for this epidemic.

\section{METHODS}

\section{STUDY AREA}

Tripura is a small north-eastern state of India, situated between latitude $22^{\circ} 57^{\prime} \mathrm{N}$ and $24^{\circ} 33^{\prime} \mathrm{N}$ and longitude $91^{\circ}$ $10^{\prime}$ and $92^{\circ} 20^{\prime} \mathrm{E}$, having a $865 \mathrm{Km}$ international border with Bangladesh from three sides and with a narrow neck border- 
ing $109 \mathrm{KM}$ with Mizoram and $53 \mathrm{Km}$ with the state of Assam. With an annual rainfall of above $2000 \mathrm{~mm}$, the climate of this state is moist and humid with temperature ranging from $21^{\circ} \mathrm{C}$ to $38^{\circ} \mathrm{C}$ in summer. Topographically, $60 \%$ of the geographical area of the state is hilly and covered by forest. Entomological surveys were made in three districts of Tripura namely West Tripura, Khowai and Dhalai districts. Among these three districts Dhalai was the most affected in the reported epidemic followed by Khowai and West Tripura respectively. Mosquitoes were collected from two villages of West Tripura, two villages of Khowai and one village of Dhalai districts. These villages are having similar ecological settings and situated in hilly forested terrain.

\section{ENTOMOLOGICAL INVESTIGATION}

House frequenting female anophelines were collected by using CDC (Centers for Disease Control and Prevention) miniature light traps. Traps were placed at a height of about 1.5 to $2 \mathrm{~m}$ from the floor adjacent to the beds where the residents usually sleep. Traps were operated from dusk to dawn and in the morning trapped mosquitoes were brought to the field laboratory. In the laboratory mosquitoes were identified to species or species complex level by using standard keys ${ }^{5}$ and kept individually after assigning an unique identification number in beam capsules containing silica gel.

Species confirmation of the morphologically identified An. dirus and An. minimus complex mosquitoes, presence of human blood in the mosquito abdomen and detection of Plasmodium species in the gland and gut of the collected anophelines were carried out by using molecular method. The head-thorax and abdomen portion of individual mosquitoes were bisected individually and homogenized in $1.5 \mathrm{ml}$ eppendorf tubes separately. To the homogenate $60 \mu \mathrm{l}$ of Phosphate Buffer Saline (PBS) was added and mixed well. The lysate was then pipetted out in FTA classic cards and allowed to dry overnight in laminar flow. The DNA was isolated from these FTA cards according to manufacturer's instruction (Whatman, Kent; UK).

For the species confirmation of the Dirus complex and Minimus complex mosquitoes, the allele specific polymerase chain reaction (ASPCR) assay based on rDNA developed by Walton et $\mathrm{al}^{6}$ and Phuck et $\mathrm{al}^{7}$ was used with minor modifications. Presence of Plasmodium infection in individual mosquitoes were detected both in head/thorax and abdomen separately for the detection of gland and gut infection by using the nested polymerase chain reaction method developed by Snounou et al. ${ }^{8}$ This rDNA based method uses genus specific primers for the first round of amplification and in the second round each parasite species is detected separately by using species specific primers. Human blood in the abdomen of blood fed specimens of Dirus and Minimus complex of mosquitoes was detected by following the protocol and primers as described by Mohanty et al. ${ }^{9}$

\section{RESULTS}

\section{ANOPHELINE DIVERSITY}

A total of 124 anopheles mosquitoes belonging to 11 species were found during the survey. Total number of $A n$. baimaii identified during the survey was 63 (about 50\%). In comparison to An. baimaii the prevalence of the other vector species from north east India was found to be less, as only $8 \%$ of the total collection was composed An. minimus (Table 1). Species identification of An. baimaii and An. minimus was confirmed by using rDNA based molecular species identification method.

\section{AVERAGE TRAP DENSITY, PLASMODIUM INFECTIONS \& HUMAN BLOOD INDEX}

In the present study, $11 \%(\mathrm{n}=7)$ of the total An. baimaii $(\mathrm{n}=63)$ were found to harbor Plasmodium falciparum (Table 2). An. baimaii was found to be present in all five villages during the epidemic. Mosquito per trap density was recorded to be lowest in a village of Jirania primary health centre (PHC), West Tripura, unaffected by the reported malaria epidemic, whereas, peak density of An. baimaii was noted in a highly affected village of Mungiakamin PHC (Table 3) under Dhalai District. Human blood index of $A n$. baimaii was recorded to be $100 \%$ in 22 nos. of blood fed specimens screened.

\section{DISCUSSION}

The earliest and only record of vector incrimination relating to human malaria in Tripura state was in 1954, when An. minimus was incriminated for malaria sporozoites. ${ }^{10}$ However, the role of the other important vector An. baimaii and of various Anopheles species present in Tripura state could not be substantiated clearly. An. minimus and An. baimaii (earlier An. dirus D) are the two primary vectors of human malaria in the north-eastern region of India. ${ }^{5}$ Earlier studies in Tripura confirmed the presence of only An. baimaii among the members of Dirus complex by using molecular methods in specimens collected from Dhalai, North Tripura, South Tripura and West Tripura districts. ${ }^{11}$

Forest Survey of India (2011) ${ }^{12}$ using satellite data of 2009 stated that $76 \%$ of the geographical area of Tripura state is under forest. About $109 \mathrm{~km}^{2}$ area under very dense forest, 4,686 $\mathrm{km}^{2}$ area under moderately dense forest and $3182 \mathrm{~km}^{2}$ under open forest. In the presence of highly efficient vector like An. baimaii, adapted to hilly forested habitats and An. minimus adapted to foothills, forest fringes and deforested hills, the malaria epidemiology of north eastern region of India is highly complex. Situation is more difficult due to the presence of several secondary vectors, whose roles are yet to be defined. The relationship of malaria transmission with forest cover and deforestation is a complex, regionally distinctive and locally specific phenomena. ${ }^{13}$ Closed or deep forested areas are the natural habitat of forest adapted hygrophilic and exophilic species like $A n$. baimaii which can spread over to the edge of the forest and fragmented forest areas during the rainy season due to the increase in humidity and breeding habitats. Human settlers within and in the vicinity of forest was considered to be a probable cause for increasing the densities of highly anthrophillic forest species like An. baimaii by providing hosts and transitory pools for egg laying. ${ }^{14}$ In contrast An. minimus, the other primary vector species of this region, adapts to near forest areas like foothills, forest fringes and 
Table 1. Anopheles mosquito species collected from five villages in Tripura

\begin{tabular}{clcc}
\hline SI. No. & \multicolumn{1}{c}{ Species name } & Nos. collected & \% composition \\
1 & An. baimaii & 63 & $50 \%$ \\
2 & An. jeyporiensis & 14 & $11 \%$ \\
3 & An. minimus & 10 & $8 \%$ \\
4 & An. varuna & 10 & $8 \%$ \\
5 & An. kochi & 8 & $6 \%$ \\
6 & An. barbirostris & 5 & $4 \%$ \\
7 & An. phili/nivipes & 5 & $3 \%$ \\
8 & An. maculates group & 4 & $2 \%$ \\
9 & An. vagus & 3 & $0.8 \%$ \\
11 & An. hyrcanus group & 1 & $0.8 \%$ \\
\hline
\end{tabular}

Table 2. Average trap density, Plasmodium infections \& human blood index in collected mosquito species

\begin{tabular}{|c|c|c|c|c|c|}
\hline \multirow[t]{2}{*}{ SI. No. } & \multirow[t]{2}{*}{ Species } & \multicolumn{2}{|c|}{ Average per trap per night density } & \multicolumn{2}{|c|}{ Plasmodium infections } \\
\hline & & Total collected (\% composition) & Per trap night & Gland & Gut \\
\hline 1 & An. baimaii & 63 & 4 & $7(n=63)$ & $2(n=56)$ \\
\hline 2 & An. minimus & 10 & 0.62 & $0(n=10)$ & $0(n=10)$ \\
\hline 3 & An. jeyporensis & 14 & 0.87 & $0(n=14)$ & $0(n=14)$ \\
\hline 4 & An. varuna & 10 & 0.62 & $0(n=10)$ & $0(n=10)$ \\
\hline 5 & An. kochi & 8 & 0.5 & $0(n=8)$ & $0(n=8)$ \\
\hline 6 & An. barbirostris & 5 & 0.31 & $0(n=5)$ & $0(n=5)$ \\
\hline 7 & An. phili/nivipes & 5 & 0.31 & $0(n=5)$ & $0(n=5)$ \\
\hline 8 & An. maculates group & 4 & 0.25 & $0(n=4)$ & $0(n=4)$ \\
\hline 9 & An. vagus & 3 & 0.18 & $0(n=3)$ & $0(n=3)$ \\
\hline 10 & An. hyrcanus group & 1 & 0.06 & $0(n=1)$ & $0(n=1)$ \\
\hline 11 & An. aconitus & 1 & 0.06 & $0(n=1)$ & $0(n=1)$ \\
\hline
\end{tabular}

deforested hilly areas. However, deforestation followed by tree crop plantation may again facilitate An. baimaii. An. baimaii a member of the Dirus complex adapts so well to rubber plantation that it was presumed to be even better than to their original tropical rain forest habitats. ${ }^{15-18} \mathrm{An}$. baimaii breeds in the shaded transient pools of a forest with good canopy cover. However, practice of jhum cultivation opens up the forest and creates favorable breeding habitat for An. minimus in the associated streams as absence of shade promotes growth of vegetation along the edges of the streams. ${ }^{19}$ Considering the scenario Guerra et al ${ }^{13}$ remarked that malaria situation in such areas are highly complex, unpredictable and not unidirectional. In the backdrop of climate change and its effects on the malaria situation may be more complex in the coming days.

For the propagation of An. baimaii, the pattern of rainfall seems to be more important as their density showed a significant positive correlation with rainfall two weeks before. ${ }^{11}$ Heavy rains can flush out the larval habitats and can also dilute the nutrients for the larvae. ${ }^{14}$ Intermittent rains not to the extent of flushing out the breeding habitats of An. baimaii and a 3 to $5^{\circ} \mathrm{C}$ higher mean temperature during the period has shortened the life cycle of the vector and reduced extrinsic cycle of the malaria parasite might have cre- ated ideal condition for vector expansion and malaria transmission. Meteorological data for Tripura from $1^{\text {st }}$ quarter of April to $4^{\text {th }}$ quarter of June, just before the onset of the epidemic, also depicted an intermittent and deficient rainfall pattern during this period in comparison to previous year. Jhum cultivation is widely practiced by the indigenous people of Tripura and a sizeable population in the hills depends on Jhum for their livelihood. ${ }^{20}$ Dhalai district which has a considerable population of Jhumias (people involved in shifting cultivation) was also recorded to be the most worst affected district in the reported epidemic. Due to population growth and for livelihood there is not only a reduction of jhum cycle from $10 / 20$ years to $2 / 3$ years but also more and more peoples are encroaching virgin dense forested areas for shifting cultivation and other activities leading to creation of high malaria endemic areas having perennial transmission near the villages of Ganganagar and Chowmanu PHC of Dhalai district.

In the present study An. baimaii was found to be the dominant species constituting $50 \%$ of the total collection (Table 3). This is much higher compared to our earlier survey where this species was found in fewer numbers Prakash et al. ${ }^{11}$ This indicates an expansion of this species during the present time under favorable condition. 
Table 3. Entomological collections using CDC light traps in malaria affected villages of Tripura

\begin{tabular}{|c|c|c|c|c|c|c|c|c|c|}
\hline \multirow{4}{*}{ SPECIES NAME } & \multicolumn{4}{|c|}{ WEST TRIPURA } & \multicolumn{4}{|c|}{ KHOWAI } & \multirow{3}{*}{$\begin{array}{c}\text { DHALAI } \\
\text { GANGANAGAR } \\
\text { PHC }\end{array}$} \\
\hline & \multirow{2}{*}{\multicolumn{2}{|c|}{$\begin{array}{l}\text { MANDAI PHC } \\
\text { HARBUNG } \\
\text { VILLAGE }\end{array}$}} & \multicolumn{2}{|c|}{ JIRONIA PHC } & \multicolumn{2}{|c|}{$\begin{array}{c}\text { TRILOSIKHAR } \\
\text { PHC }\end{array}$} & \multicolumn{2}{|c|}{$\begin{array}{c}\text { MUNGIAKAMIN } \\
\text { PHC }\end{array}$} & \\
\hline & & & \multicolumn{2}{|c|}{ SINAI VILLAGE } & \multicolumn{2}{|c|}{$\begin{array}{l}\text { UDAN MAIDAN } \\
\text { VILLAGE }\end{array}$} & \multicolumn{2}{|c|}{$\begin{array}{l}\text { HUMBAI } \\
\text { VILLAGE }\end{array}$} & \\
\hline & No. & $\begin{array}{l}\text { PER } \\
\text { TRAP }\end{array}$ & NO. & $\begin{array}{l}\text { PER } \\
\text { TRAP }\end{array}$ & NO. & $\begin{array}{l}\text { PER } \\
\text { TRAP }\end{array}$ & NO. & $\begin{array}{l}\text { PER } \\
\text { TRAP }\end{array}$ & NO \\
\hline An. aconitus & - & - & 1 & 0.25 & - & - & - & - & - \\
\hline An. barbirostris & 1 & 0.3 & 1 & 0.25 & 1 & 0.3 & 3 & 1 & - \\
\hline An. baimaii & 12 & 4 & 2 & 0.5 & 5 & 1.7 & 37 & 9.2 & 7 \\
\hline $\begin{array}{l}\text { An.hyrcanus } \\
\text { group }\end{array}$ & - & - & 1 & 0.25 & - & - & - & - & - \\
\hline An. jeyporiensis & - & - & 7 & 1.8 & - & - & 8 & 2.7 & - \\
\hline An. kochi & - & - & 3 & 0.8 & - & - & 5 & 1.7 & - \\
\hline An. minimus & - & - & - & - & 9 & 3 & - & - & 1 \\
\hline $\begin{array}{l}\text { An. maculates } \\
\text { group }\end{array}$ & 2 & 0.7 & 1 & 0.25 & - & - & - & - & - \\
\hline An. nivipes & - & - & 5 & 1.2 & - & - & 1 & 0.3 & - \\
\hline An. varuna & - & - & - & - & - & - & - & - & 1 \\
\hline An. vagus & - & - & 3 & 0.8 & - & - & 1 & 0.3 & - \\
\hline
\end{tabular}

PHC - primary health centre

Our study revealed $11 \%$ An. baimaii (n=63) infected with Plasmodium falciparum by using molecular assay indicating that the species is playing a major role in the present epidemic (Table 2). Sporozoite rates of $14 \%$ were reported in An. dirus s.l. from forested areas of Myanmar. ${ }^{21}$ In a highly anthropophilic species like An. baimaii, a small population of the vector can maintain a high level of transmission. ${ }^{15}$ The recorded 100\% human blood index in An. baimaii collected during the epidemic period is in agreement with its pivotal role in the epidemic.

The importance of An. baimaii as a vector of human malaria in north-eastern India was first noted when malaria persisted even after achieving excellent control of An. minimus by using DDT as indoor residual spray. ${ }^{17}$ Exclusively exophilic nature of this species makes it difficult to control by using Indoor residual spray. ${ }^{14}$ DDT spray coverage was found to be poor in Tripura state. Spray virtually started from the month of June in Tripura in the epidemic year and hence before its completion the epidemic already appeared. Although DDT might be comparatively less effective for this species, still the spray could have reduced the man-vector contact to some extent and is effective against the other important vector An. minimus from NE India. A study from Bangladesh shown that An. baimaii avoid sprayed huts by biting more in outdoors. ${ }^{22}$ In Thailand DDT treated huts showed a decline of $79.4 \%$ in attempted blood feeding by An. dirus s.l. ${ }^{23}$ Looking into the biting habit of An. baimaii in north-eastern India a sizeable malaria transmission risk exists during the pre-bed time (18.00-21.00 hours $)^{24}$ that can be avoided by using insecticide treated nets supplemented with repellent creams as reported by Prakash et al. ${ }^{2}$ Whereas in case of An. minimus effective control can be possible by using ITNs alone as only a small proportion of this species bites before 21:00 hours. ${ }^{25}$ Long lasting insectici- dal nets (LLINs) were used in Tripura distributed by Public Health Department from 2009-12. However, coverage was not full and they were distributed without studying community acceptance of using bed nets, bed net size, sleeping pattern etc. On the basis of these findings, considering the hilly forested terrain and remoteness of many villages inhabited by these vectors in Tripura and for the difficulty of implementing vector control measures in time, scaling up of LLINs seems to be highly essential.

Behavior of An. baimaii like avoiding contact with the sprayed walls of the human dwellings and its early biting habit is a major constraint for achieving complete vector control even after using IRS and ITN/LLIN in combination. In this context, the situation in Tripura seems further worsened due to the inadequate implementation of these routine vector control measures.

As evidenced by our study involvement of An. baimaii in Tripura epidemic does not augur well for future if appropriate control measures are not undertaken as it is an exclusively anthropophilic species found in the forested areas of north east India.

\section{CONCLUSIONS}

To summarize the present situation, we can infer that human settlements in deep forested areas combined with favorable environmental condition and inadequate vector control help in the expansion of densities of forest adapted species like An. baimaii leading to an epidemic situation. Considering the rapid topographical changes in Tripura due to deforestation and commercial plantation coupled with the effect of climatic changes, the malaria situation in Tripura will be unpredictable in coming years. 


\section{COMPETING INTERESTS}

\section{ACKNOWLEDGEMENTS}

The Authors acknowledge the support provided by Tripura State Health Authorities during the survey. Technical assistance provided by Mr. Lakhyajit Borah is highly acknowledged.

\section{FUNDING}

The Authors are grateful to Indian Council of Medical Research for funding the study.
The authors completed the Unified Competing Interest form at http://www.icmje.org/coi_disclosure.pdf (available upon request from the corresponding author), and declare no conflicts of interest.

\section{CORRESPONDENCE TO:}

Dibya Ranjan Bhattacharyya Ph.D

Scientist "F"

ICMR-Regional Medical Research Centre, NE

P. Box-105; Pin: 786001

Dibrugarh Assam

India

drbhattacharyya@yahoo.com 


\section{REFERENCES}

1. Mohapatra PK, Prakash A, Bhattacharyya DR, Mahanta J. Malaria situation in north-eastern region of India. Icmr Bull. 1998;28:21-30.

2. Prakash A, Bhattacharyya DR, Mohapatra PK, Barua A, Phukan A, Mahanta J. Malaria control in a forest camp in an oil exploration area of upper Assam. Natl Med J India. 2003;16:135-18.

3. JanaKara B, WajihullahSahi B, Dev V, Curtis CF, Sharma VP. Deltramethrin impregnated bed nets against Anopheles minimus transmitted malaria in Assam, India. Am J Trop Med Hyg. 1995;98:73-83.

4. Misra BG, Dhar SK. Malaria in Tripura state. Indian J Malariol. 1955;9:111-123.

5. Das SC, Bhuayan M, Baruah I, Talukdar PK. Mosquito survey in Tripura. Indian J Malariol. 1991;28:129-134.

6. Walton C, Handley JM, Kuvangkadilok C, et al. Identification of five species of the Anopheles dirus complex from Thailand, using allele-specific polymerase chain reaction. Med Vet Entomol. 1999;13(1):24-32. doi:10.1046/j.1365-2915.1999.0014 2.x

7. Phuc HK, Ball AJ, Son L, et al. Multiplex PCR assay for malaria vector Anopheles minimus and four related species in the Myzomia series from South east Asia. Med Vet Entomol. 2003;17(4):423-428. doi:10.11 11/j.1365-2915.2003.00462.x

8. Snounou G, Viriyakosol S, Jarra W, et al. High sensitivity of detection of human malaria parasites by the use of nested polymerase chain reaction. $\mathrm{Mol}$ Biochem Parasitol. 1993;61(2):315-320. doi:10.1016/0 166-6851(93)90077-b

9. Mohanty A, Kar SK, Mohapatra N, et al. Multiplex PCR assay for the detection of Anopheles fluviatilis species complex, human host preference, sporozoite presence, using a unique mosquito processing method. Am J Trop Med Hyg. 2007;76(5):837-843. do i:10.4269/aitmh.2007.76.837

10. Das BP, Rajagopal R, Akiyama J. Pictorial key to the species of Indian anopheline mosquitoes. Zoology. 1990;2:131-162.

11. Prakash A, Sarma DK, Bhattacharyya DR, et al. Spatial distribution and r-DNA second internal transcribed spacer characterization of Anopheles dirus (Diptera: Culicidae) complex species in northeast India. Acta Trop. 2010;114(1):49-54. doi:10.1016/ j.actatropica.2010.01.003
12. Forest survey of India report. Published 2011. Accessed June 7, 2017. https://www.fsi.nic.in

13. Guerra CA, Snow RW, Hay SI. A global assessment of closed forests, deforestation and malaria risk. Ann Trop Med Parasitol. 2006;100(3):189-204. doi:10.117 9/136485906x91512

14. Prakash A, Bhattacharyya DR, Mohapatra PK, Mahanta J. Studies on bionomics of Anopheles dirus Peyton and Harrison, 1997 (Diptera: Culicidae) in Assam, India. Proceedings of the second Symposium on Vectors \& Vector Borne Diseases. Published online 1997:236-246.

15. Obsomer V, Defourny P, Coosemans M. The Anopheles dirus complex: spatial distribution and environmental drivers. Malar J. 2007;6(26). doi:10.11 $\underline{86 / 1475-2875-6-26}$

16. Rosenberg R, Andre RG, Ngampatom S, Hatz C, Burge R. A stable, oligosymptomatic malaria focus in Thailand. Trans R Soc Trop Med Hyg. 1990;84(1):14-21. doi:10.1016/0035-9203(90)9036 6-m

17. Yasuoka J, Levins R. Impact of deforestation and agriculture development on Anopheline ecology and malaria epidemiology. Am J Trop Med Hyg. 2007;76(3):450-460. doi:10.4269/ajtmh.2007.76.450

18. Petney T, Sithithaworn P, Satrawaha R, et al. Potential malaria reemergence, northeastern Thailand. Emerg Infect Dis. 2009;15(8):1330-1331. do i:10.3201/eid1508.090240

19. Rao TR. The Anophelines of India. Malaria Research Centre; 1984.

20. Das S, Das M. Shifting cultivation in Tripura-A critical analysis. Journal of Agriculture and Life Sciences. 2014;1:48-54.

21. Prakash A, Bhattacharyya DR, Mohapatra PK, Mahanta J. Investigation on malaria vectors and mosquito fauna in South Tripura district, Tripura state. Indian J Malariol. 1998;35:151-19.

22. Kyi KM. Malaria vectors in Burma 2. Anopheles balbacensis balbacensis Baisas, 1936. Union Burma J Life Sci. 1970;3:217-225.

23. Malaithong N, Polsomboon S, Poolprasert P, et al. Human-landing patterns of Anopheles dirus sensu lato (Diptera: Culicidae) in experimental huts treated with DDT or Deltamethrin. J Med Entomol. 2010;47(5):823-832. doi:10.1093/jmedent/47.5.823 
24. Prakash A, Bhattacharyya DR, Mohapatra PK, Mahanta J. Malaria transmission risk by the mosquito (formerly known as An. dirus species D) at different hours of night in north-east India. Med Vet Entomol. 2005;19(4):423-427. doi:10.1111/j.1365-2915.2005.00 592.x
25. Dev V. Anopheles minimus: its bionomics and role in the transmission of malaria in Assam, India. Bull World Health Organ. 1996;74:61-66. 